\title{
Increased chemiluminescence activity of blood neutrophilic granulocytes from patients with Graves' disease
}

Margarita A. Dudina, Sergey A. Dogadin, Andrey A. Savchenko

Krasnoyarsk State Medical University named after prof. V. F. Voino-Yasenetsky

Institute for Medical Problems of the North, Siberian Division, Russian Academy of Medical Sciences

Krasnoyarsk regional clinical hospital, Krasnoyarsk, Russian Federation

\section{OBJECTIVES}

The role of neutrophilic granulocytes in pathogenesis of Graves' disease

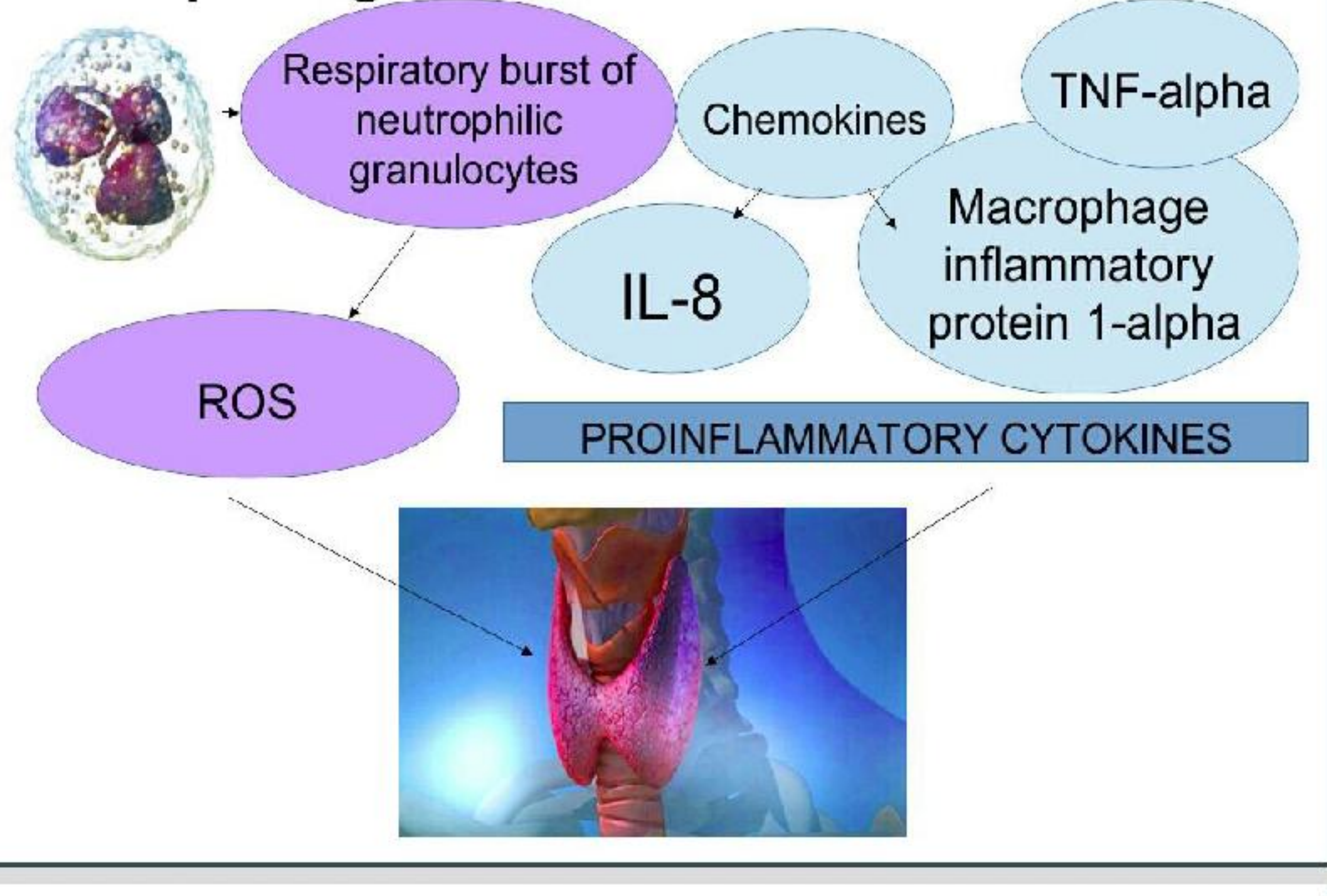

Aim: to investigate chemiluminescent $(C L)$ activity of blood neutrophilic granulocytes in patients with Graves disease (GD).

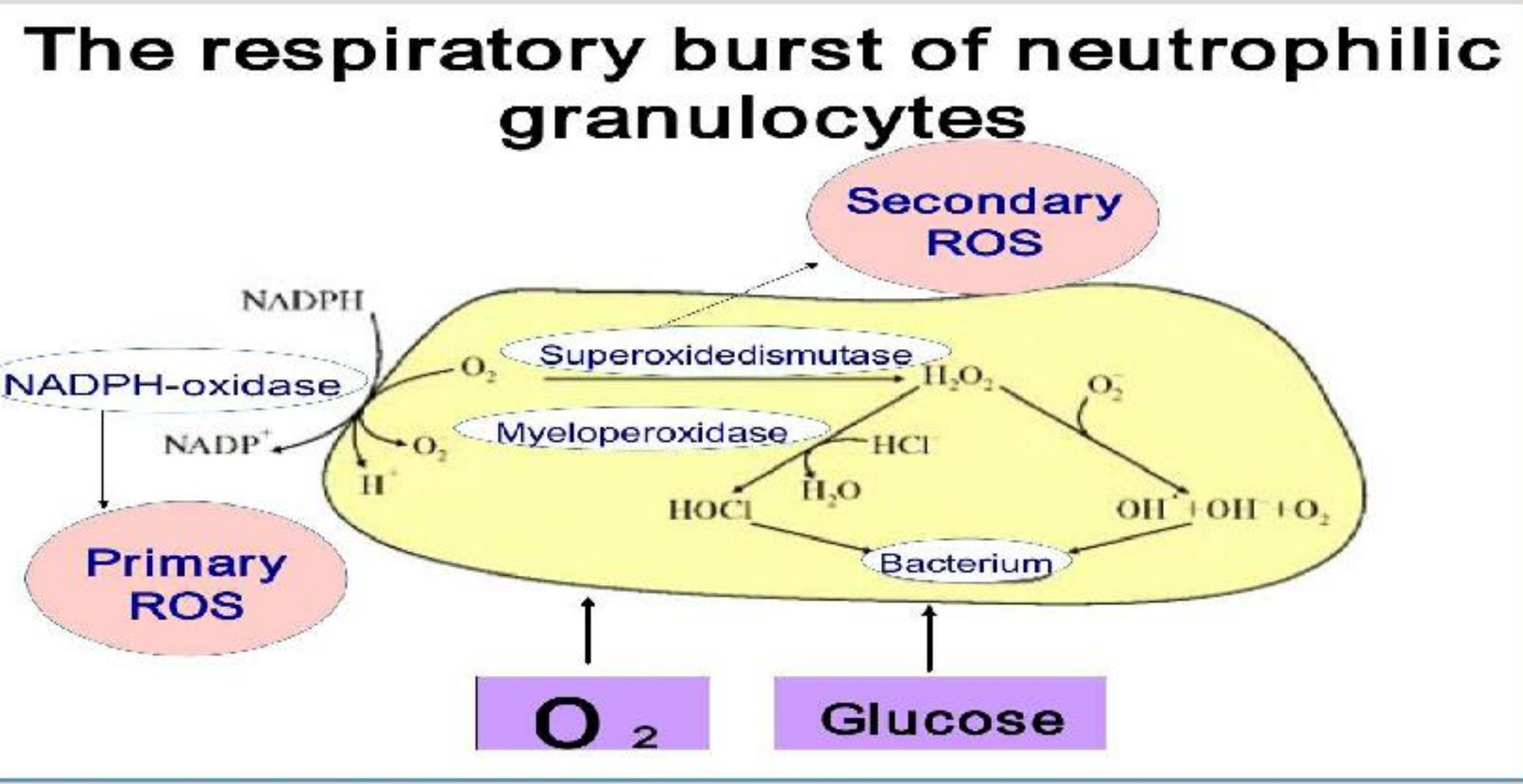

METHODS

\section{Study design}

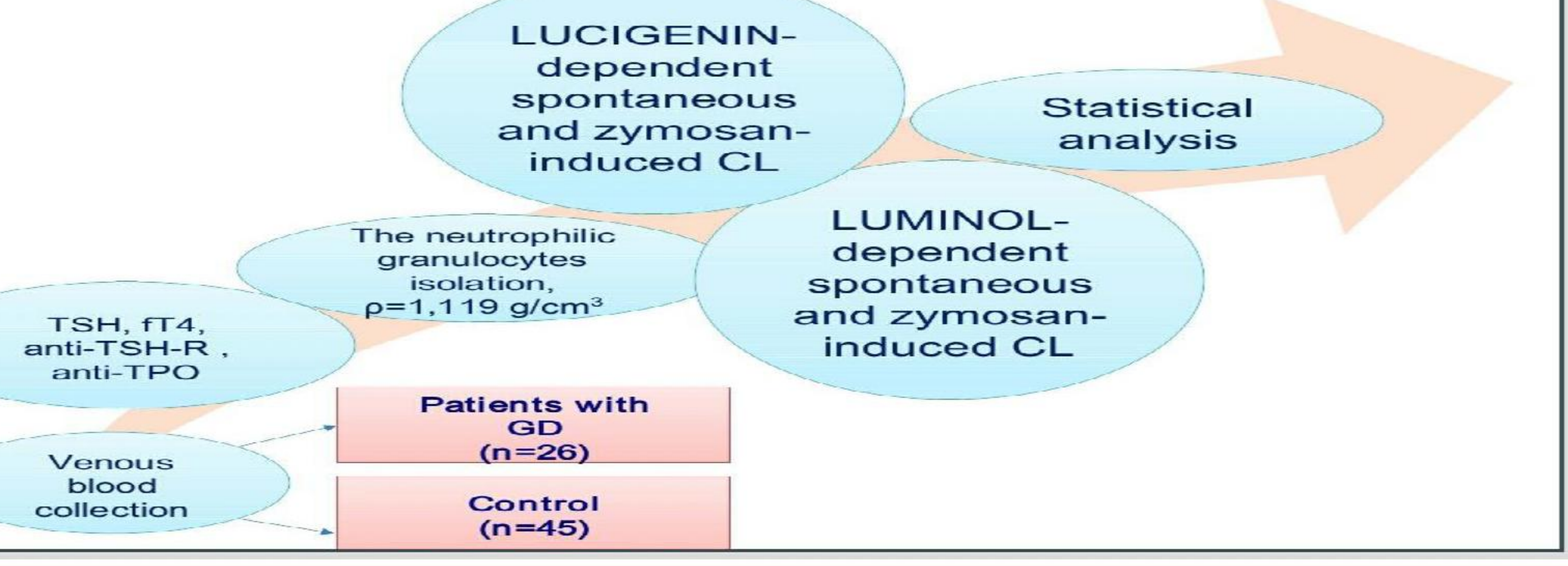

Twenty six women, mean age $40,7 \pm 13,2$, with manifestation of GD up to antithyroid therapy and 30 sex- and age-matched healthy subjects were examined. Serum thyroid hormone concentrations were measured by RIA. The median of TSH, fT4, anti-TSHR and anti-TPO concentration in GD patient were $0,17(0,01 ; 0,72) \mathrm{mU} / \mathrm{l}, 17,9(13.1 ; 30,3)$ $\mathrm{pmol} / \mathrm{l}, 16,81 \mathrm{IU} / \mathrm{l}$ and $374,0(223,0 ; 817,4) \mathrm{IU} / \mathrm{ml}$ respectively. The neutrophilic granulocytes were isolated from heparinized whole blood by double density gradient centrifugation in the ficoll-urografin: $\rho=1.077 \mathrm{~g} / \mathrm{cm}^{3}$ for lymphocytes separation, $\rho=1,119$ $\mathrm{g} / \mathrm{cm}^{3}$ for the neutrophils isolation. The spontaneous and zymosan-induced $(\mathrm{ZiCL})$ was studied for 90 minutes on 36-channel chemiluminescent analyzer "CL3606". The following characteristics have been identified: time to maximum (Tmax), maximum intensity value (Imax), reflecting the maximum reactive oxygen species (ROS) level synthesis and the area under the curve (S), describing total synthesis of ROS during $90 \mathrm{~min}$. of the study.

\section{RESULTS}

Fig. 1. The indicators of spontaneous (a) and zymosan-induced (b) CL in patient with GD ( $\mathrm{n}=26$ ) and control group ( $\mathrm{n}=45$ ).

a) The indicators of spontaneous CL in patients with GD and control group

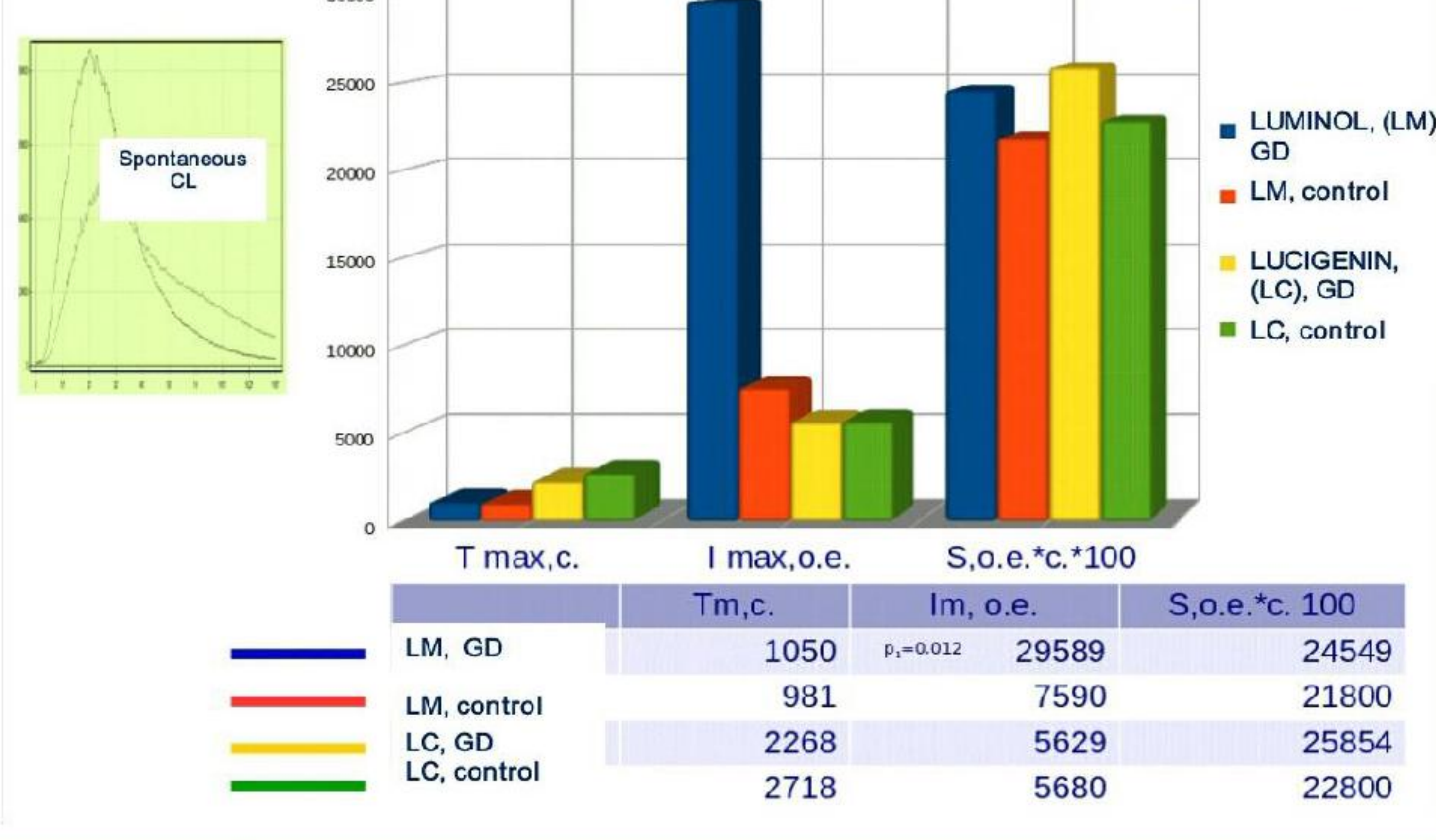

b) The indicators of zymosan-induced ( $\mathrm{ZiCL})$

in patients with GD and control group

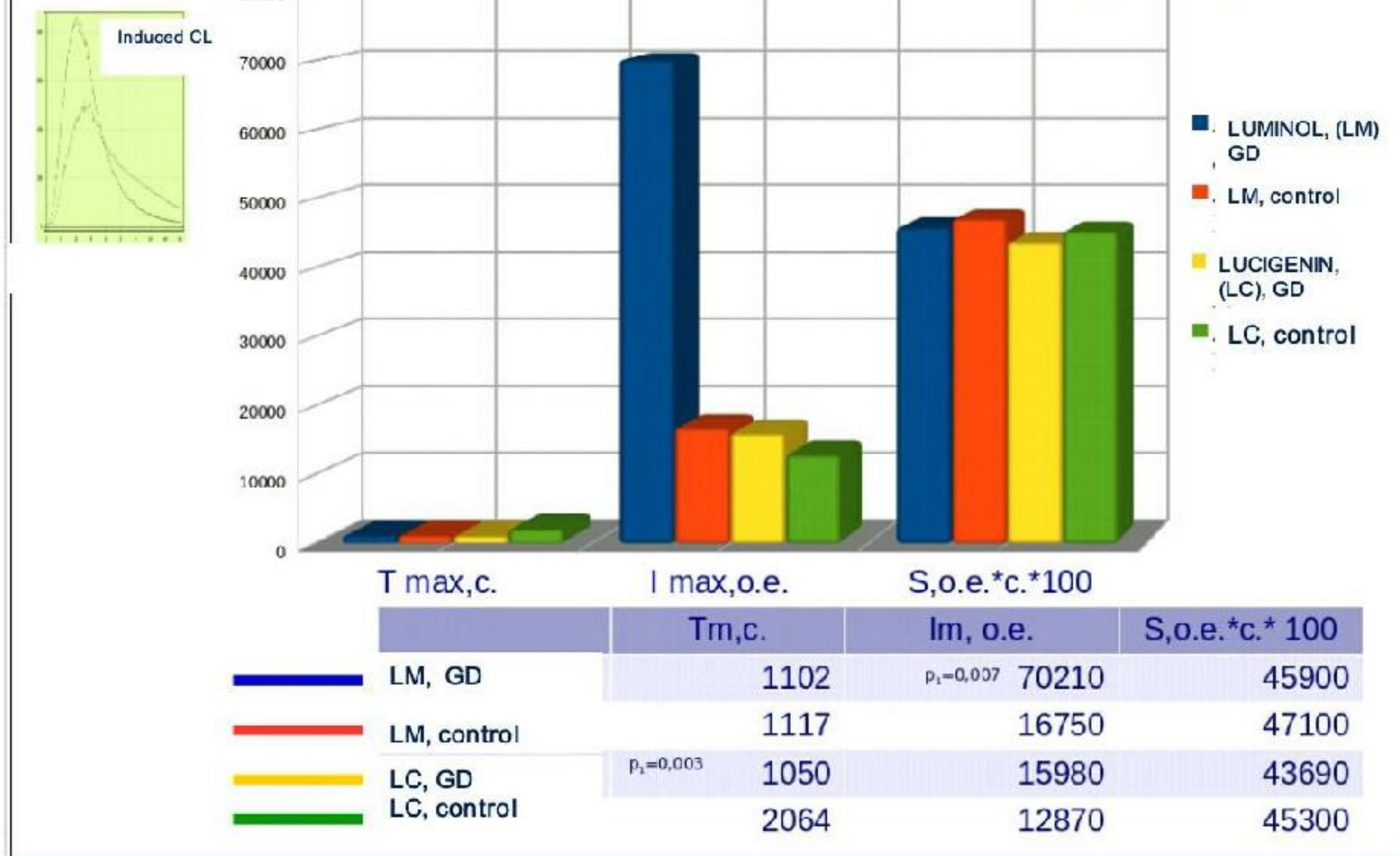

Fig. 2. The pathological interaction between autoimmune thyroid marker and $\mathrm{CL}$ indicators in patients with $\mathrm{GD}(\mathrm{n}=26)$.

The Spearmen correlation in anti-TPO level $\& \mathrm{CL}$ indicators of blood neutrophils in patients with $G D$

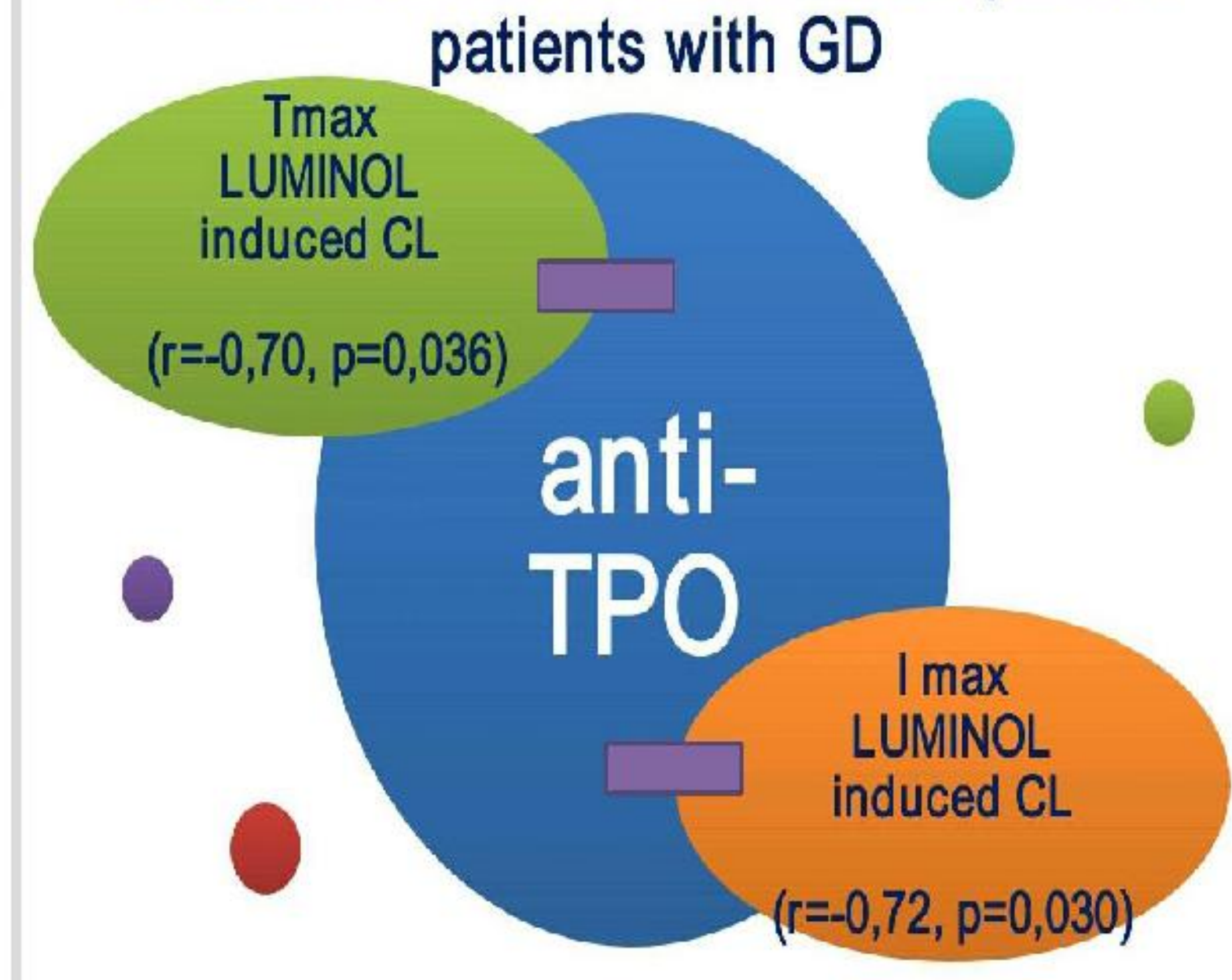

Fig. 3. The interaction between thyroid hormone and $C L$ indicators in healthy controls $(n=45)$.
The Spearmen correlation in TSH level \& $\mathrm{CL}$ indicators of blood neutrophils in



The Spearmen correlation in fT4 level \& $\mathrm{CL}$ indicators of blood neutrophils in

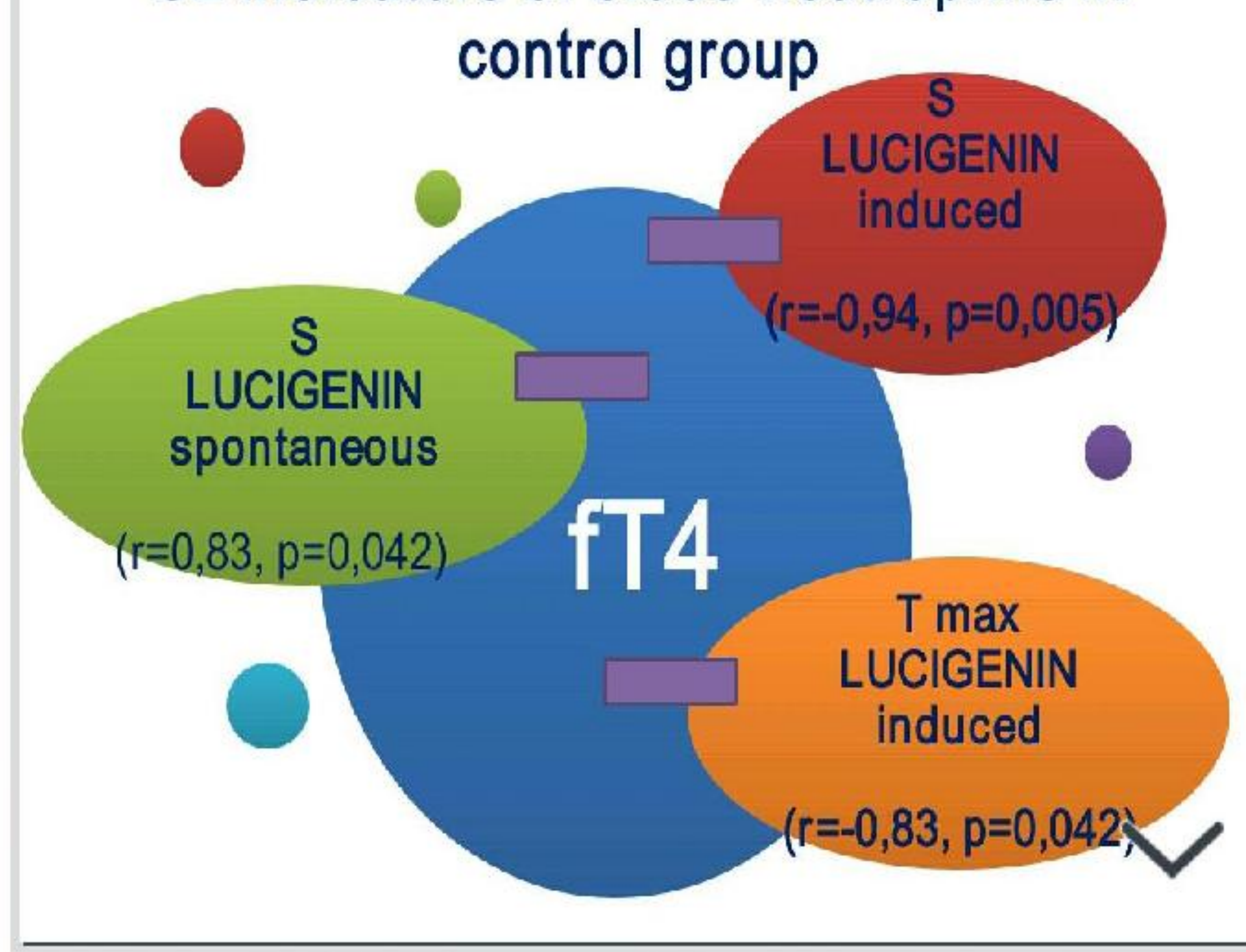

\section{CONCLUSIONS}

Hyperactivity and reactivity of peripheral blood neutrophils in GD certify by respiratory explosion in spontaneous and induced of secondary ROS level synthesis. Enhanced excitability to phagocytosis-related stimuli can mediate the autoimmune mechanism in GD.

\section{References}

1) UrazovaOI, Kravets EB, Novitsky VV et al. Clin and experimental thyroidologiy 2007; 3 (4) :49-53.

2) Savchenko AA, Borisov AG Novosibirsk: Science 2012263 p

3) Krieger CC, Neumann S, Place RF et al. J Clin Endocrinol Metab 2015;100(3):1071-1077. 